\title{
On the Total Edge Irregularity Strength of Disjoint Union of Graphs
}

\author{
Martin Bača ${ }^{1}$, Joe Ryan ${ }^{2}$ and Andrea Semaničová-Feňovčíková ${ }^{3 *}$ \\ ${ }^{1}$ Technical University of Košice, Faculty of Mechanical Engineering, Department of Applied Mathematics and Informatics, Košice, Slovakia \\ 2 University of Newcastle, School of Electrical Engineering and Computer Science, Callaghan NSW, Australia
}

\begin{abstract}
For a simple graph $G=(V(G), E(G)$ ), a total labeling $f: V(G) \cup E(G)$ $\rightarrow\{1,2, \cdots, k\}$ is called $k$-labeling. The weight of an edge $x y$ in $G$, denoted by $w t_{f}(x y)$, is the sum of the edge label itself and the labels of end vertices $x$ and $y$, i.e. $w t_{f}(x y)=f(x)+f(x y)+f(y)$. A total $k$-labeling is defined to be an edge irregular total $k$-labeling of the graph $G$ if for every two different edges $x y$ and $x^{\prime} y^{\prime}$ there is $w t_{f}(x y) \neq w t_{f}\left(x^{\prime} y^{\prime}\right)$. The minimum $k$ for which the graph $G$ has an edge irregular total $k$-labeling is called the total edge irregularity strength of $G$, denoted by $\operatorname{tes}(G)$. In this paper, we estimate the upper bound of the total edge irregularity strength of disjoint union of multiple copies of a graph and we prove that this upper bound is tight.
\end{abstract}

Keywords: Edge irregular total labeling, vertex irregular total labeling, totally irregular total labeling, total edge irregularity strength, total vertex irregularity strength.

\section{Introduction}

The concept of labeling of graphs has gained immense popularity in the area of graph theory. This popularity is not only due to mathematical challenges of graph labelings but also to the wide range of applications that graph labelings offer to other branches of science, for instance, X-ray crystallography, coding theory, cryptography (secret sharing schemes), astronomy, circuit design and communication design [11], [12].

Informally, by a graph labeling we will mean an assignment of integers to elements of a graph, such as vertices, or edges, or both, subject to some specified conditions. These conditions are usually expressed on the basis of the values (called weights) of some evaluating function. In our case, the evaluating function will be simply to produce partial sums of the labeled elements of the graph. The partial sums will be either a set of vertex weights, obtained for each vertex by adding all the labels of a vertex and its adjacent edges, or a set of edge weights, obtained for each edge by adding the labels of an edge and its endpoints.

\section{Definitions and Known Results}

Let $G$ be a connected, simple and undirected graph with vertex set $V(G)$ and edge set $E(G)$. For an edge $k$-labeling $\psi: E(G) \rightarrow\{1,2, \cdots, k\}$ the associated weight of a vertex $x \in V(G)$ is

$$
w_{\psi}(x)=\sum \psi(x y),
$$

where the sum is over all vertices $y$ adjacent to $x$. 
Chartrand, Jacobson, Lehel, Oellermann, Ruiz and Saba in [15] introduced edge $k$-labeling $\psi$ of a graph $G$ such that $w_{\psi}(x) \neq w_{\psi}(y)$ for all vertices $x, y \in V(G)$ with $x \neq y$. Such labelings were called irregular assignments and the irregularity strength $s(G)$ of a graph $G$ is known as the minimum $k$ for which $G$ has an irregular assignment using labels at most $k$. The irregularity strength $s(G)$ can be interpreted as the smallest integer $k$ for which $G$ can be turned into a multigraph $G^{\prime}$ by replacing each edge by a set of at most $k$ parallel edges, such that the degrees of the vertices in $G^{\prime}$ are all different.

Finding the irregularity strength of a graph seems to be hard even for graphs with simple structure, see $[5,7,13,16,17,22,24,26]$.

The lower bound of the irregularity strength is given by the following inequality

$s(G) \geq \max _{1 \leq i \leq \Delta(G)} \frac{i-1+n_{i}}{i}$,

where $n_{i}$ denotes the number of vertices of degree $i$ and $\Delta(G)$ is the maximum degree of graph $G$. Moreover in the case of $r$-regular graph it reduce to the following form

$$
s(G) \geq \frac{r-1+n}{r} .
$$

Nierhoff [26] proved that for any graph $G \neq K_{3}$ with $p$ vertices and no component of order at most 2 , the irregularity strength is at most $p-1$. It gives an upper bound for $s(G)$.

Motivated by irregular assignments, Bača, Jendrol, Miller and Ryan in [8] defined a vertex irregular total $k$-labeling of a graph $G$ to be a total labeling of $G, f: V(G) \cup E(G) \rightarrow\{1,2, \cdots, k\}$, such that the vertex-weights

$$
w t_{f}(x)=f(x)+\sum_{x y \in E(G)} f(x y)
$$

are different for all vertices, that is, $w t_{f}(x) \neq w t_{f}(y)$ for all different vertices $x, y \in V(G)$. The total vertex irregularity strength of a graph $G$, denoted by $\operatorname{tvs}(G)$, is the minimum $k$ for which $G$ has a vertex irregular total $k$-labeling.

It is easy to see that the irregularity strength $s(G)$ of a graph $G$ is defined only for graphs containing at most one isolated vertex and no connected component of order 2.

On the other hand, the total vertex irregularity strength $\operatorname{tvs}(G)$, is defined for every graph $G$. Thus for graphs with no component of order $\leq 2$, tvs $(G) \leq s(G)$.

In [8] several bounds and exact values of $\operatorname{tvs}(G)$ were determined for different types of graphs (in particular for stars, cliques and prisms).

The bounds for the total vertex irregularity strength are given by the following theorem.

\section{Theorem 1. [8]}

Let $G$ be a $(p, q)$-graph with minimum degree $\delta(G)$ and maximum degree $\Delta(G)$. Then

$\left\lceil\frac{p+\delta(G)}{\Delta(G)+1}\right\rceil \leq t v s(G) \leq p+\Delta(G)-2 \delta(G)+1$.

A better lower bound determined Nurdin, Baskoro, Salman and Gaos as follows

\section{Theorem 2. [28]}

Let $G$ be a connected graph having $n_{i}$ vertices of degree $i, \quad i=\delta(G), \delta(G)+1, \delta(G)+2, \cdots, \Delta(G)$, where $\delta(G)$ and $\Delta(G)$ are the minimum and the maximum degree of $G$, respectively. Then

$$
\begin{aligned}
& \operatorname{tvs}(G) \geq \max \left\{\left\lceil\frac{\delta(G)+n_{\delta(G)}}{\delta(G)+1}\right\rceil,\right. \\
& \left.\left\lceil\frac{\delta(G)+n_{\delta(G)}+n_{\delta(G)+1}}{\delta(G)+2}\right\rceil, \cdots,\left\lceil\frac{\delta(G)+\sum_{i=\delta(G)}^{\Delta(G)} n_{\mathrm{i}}}{\Delta(G)+1}\right\rceil\right\} .
\end{aligned}
$$

For a regular hamiltonian $(p, q)$-graph $G$ it is proved in [8] that $\operatorname{tvs}(G) \leq[(p+2) / 3]$. Moreover there is proved the following upper bound.

\section{Theorem 3. [8]}

Let $G$ be a $(p, q)$-graph with maximum degree $\Delta(G)$ and no component of order $\leq 2$. Then

$$
t v s(G) \leq p-1-\left\lfloor\frac{p-2}{\Delta(G)+1}\right\rfloor \text {. }
$$

These results were then improved by Przybylo in 
[31] who proved that $t v s(G)<32 p / \delta(G)+8$ in general and $t v s(G)<8 p / r+3$ for $r$-regular graphs. This was then improved in [6] that

$\operatorname{tvs}(G) \leq 3\left\lceil\frac{p}{\delta(G)}\right\rceil+1 \leq \frac{3 p}{\delta(G)}+4$.

Recently Majerski and Przybylo in [23] based on a random ordering of the vertices proved that if $\delta(G) \geq n^{0.5} \ln n$ then

$$
\operatorname{tvs}(G) \leq \frac{(2+o(1)) p}{\delta(G)}+4 .
$$

The exact values of the total vertex irregularity strength for complete bipartite graphs and for wheels, fans, suns and friendship graphs can be found in [33] and [32]. Nurdin et al. determined exact values of tws for several types of trees and for disjoint union of paths in [28], [29] and [30]. Ahmad and Bača [1] found exact values of tvs for Jahangir graphs and circulant graphs.

For a total $k$-labeling $f: V(G) \cup E(G) \rightarrow\{1,2, \cdots, k\}$ of a graph $G$ the associated edge-weight of an edge $x y \in E(G)$ is defined as

$$
w t_{f}(x y)=f(x)+f(x y)+f(y) .
$$

In [8] is defined the total labeling $f$ to be an edge irregular total $k$-labeling of the graph $G$ if for every two different edges $x y$ and $x^{\prime} y^{\prime}$ of $G$ one has $w t_{f}(x y) \neq w t_{f}\left(x^{\prime} y^{\prime}\right)$. The total edge irregularity strength of a graph $G$, denoted by tes $(G)$, is defined as the minimum $k$ for which $G$ has an edge irregular total $k$-labeling.

In [8] we can find a lower bound of the total edge irregularity strength in the following form.

\section{Theorem 4. [8]}

Let $G=(V(G), E(G))$ be a graph with vertex set $V(G)$, a non-empty edge set $E(G)$ and with maximum degree $\Delta(G)$. Then

$$
\operatorname{tes}(G) \geq \max \left\{\left\lceil\frac{|E(G)|+2}{3}\right\rceil,\left\lceil\frac{\Delta(G)+1}{2}\right\rceil\right\} .
$$

Also there are determined the exact values of the total edge irregularity strength for paths, cycles, stars, wheels and friendship graphs.
Recently Ivančo and Jendrol' [19] proved that for any tree $T$ the total edge irregularity strength is

$$
\operatorname{tes}(T)=\max \left\{\left\lceil\frac{|E(T)|+2}{3}\right\rceil,\left\lceil\frac{\Delta(T)+1}{2}\right\rceil\right\} .
$$

Moreover, they posed the following conjecture.

\section{Conjecture 1. [19]}

Let $G$ be a graph different from $K_{5}$. Then

$$
\operatorname{tes}(G)=\max \left\{\left\lceil\frac{|E(G)|+2}{3}\right\rceil,\left\lceil\frac{\Delta(G)+1}{2}\right\rceil\right\} .
$$

This conjecture has been verified for complete graphs and complete bipartite graphs in [20] and [21], for the Cartesian, categorical and strong products of two paths in $[25,3,2]$, for toroidal fullerenes in [9], for the categorical product of two cycles in [4], for generalized Petersen graphs in [18], for generalized prisms in [10], for corona product of a path with certain graphs in [27] and for large dense graphs with $(|E(G)|+2) / 3 \leq(\Delta(G)+1) / 2$ in [14].

In this paper we investigate the existence of the edge irregular total labelings. We estimate the upper bound of the total edge irregularity strength of disjoint union of multiple copies of a graph and determine the exact value of the total edge irregularity strength for disjoint union of two copies of $C_{4}$.

\section{An Upper Bound of the Total Edge Irregularity Strength}

In this section, we are studying the total edge irregularity strength of a disjoint union of $m$ copies of an arbitrary graph $G$, denoted by $m G$. Next theorem gives an upper bound of the $\operatorname{tes}(m G)$.

\section{Theorem 5 .}

Let $G$ be a graph with $\operatorname{tes}(G)=k$. Then the total edge irregularity strength of the disjoint union of $m \geq 1$ copies of $G$ is

$$
\text { tes }(m G) \leq m k \text {. }
$$

\section{Proof.}

Let $G=(V(G), E(G))$ be a graph with $\operatorname{tes}(G)=k$ 
and let $f$ be the corresponding edge irregular total $k$-labeling of the graph $G$. It means that for every couple of edges $e_{i}, e_{j} \in E(G), i \neq j$, the edge-weights are distinct, i.e.

$$
w t_{f}\left(e_{i}\right) \neq w t_{f}\left(e_{j}\right) .
$$

Let us denote the edges of $G$ by the symbols $e^{1}, e^{2}, \cdots, e^{|E(G)|}$ such that

$$
w t_{f}\left(e^{j}\right)<w t_{f}\left(e^{j+1}\right)
$$

for $j=1,2, \cdots,|E(G)|-1$.

For every vertex $v$ in $G$, we denote by symbol $v_{i}$ the corresponding vertex of $v$ in the $i$-th copy of $G$, in $m G$.

Analogously, let $u_{i} v_{i}$ denote the corresponding edge of $u v$ in the the $i$-th copy of $G$, in $m G$.

We define a labeling $g$ of $m G$ in the following way

$$
g\left(v_{i}\right)=m(f(v)-1)+i,
$$

for $v \in V(G), i=1,2, \cdots, m$ and

$$
g\left(u_{i} v_{i}\right)=m f(v)+1-i,
$$

for $u v \in E(G), i=1,2, \cdots, m$.

It is easy to see that the labeling $g$ assigns the labels $1,2, \cdots, m k$ to the edges and to the vertices of $m G$.

For the edge-weight of $e_{i}^{j}=u_{i} v_{i} \in E(m G)$, $i=1,2, \cdots, m, j=1,2, \cdots,|E(G)|$, under the labeling $g$ we have

$$
\begin{aligned}
w t_{g}\left(e_{i}^{j}\right)=w t_{g} & \left(u_{i} v_{i}\right) \\
& =g\left(u_{i}\right)+g\left(v_{i}\right)+g\left(u_{i} v_{i}\right) \\
& =\left(m\left(f\left(u_{i}\right)-1\right)+i\right) \\
& +\left(m\left(f\left(v_{i}\right)-1\right)+i\right) \\
& +\left(m f\left(u_{i} v_{i}\right)+1-i\right) \\
& =m\left(f\left(u_{i}\right)+f\left(v_{i}\right)+f\left(u_{i} v_{i}\right)-2\right) \\
& +1+i \\
& =m w t_{f}(u v)-2 m+1+i \\
& =m w t_{f}\left(e^{j}\right)-2 m+1+i .
\end{aligned}
$$

Let us consider the edge-weights of the edges $e_{1}^{j}, e_{2}^{j}, \cdots, e_{m}^{j}, j=1,2, \cdots,|\mathrm{E}(\mathrm{G})|$, under the labeling $g$ : $w t_{g}\left(e_{1}^{j}\right)=m w t_{f}\left(e^{j}\right)-2 m+2$,

$w t_{g}\left(e_{2}^{j}\right)=m w t_{f}\left(e^{j}\right)-2 m+3$,

$w t_{g}\left(e_{m}^{j}\right)=m w t_{f}\left(e^{j}\right)-m+1$.

Using the inequality (1) we get that for $j=1,2, \cdots,|E(G)|-1$, it holds

$w t_{f}\left(e^{j}\right) \leq w t_{f}\left(e^{j+1}\right)-1$.

Multiplying this inequality by $m, m \geq 1$, we get

$m w t_{f}\left(e^{j}\right) \leq m w t_{f}\left(e^{j+1}\right)-m<m w t_{f}\left(e^{j+1}\right)-m+1$.

After adding the constant $1-m$ to the both sides in the previous inequality we have

$m w t_{f}\left(e^{j}\right)-m+1<m w t_{f}\left(e^{j+1}\right)-2 m+2$

which means that

$w t_{g}\left(e_{m}^{j}\right)<w t_{g}\left(e_{1}^{j+1}\right)$

for $j=1,2, \cdots,|E(G)|-1$. This completes the proof.

The upper bound of the total edge irregularity strength in Theorem 5 is tight as can be seen from the next theorem.

\section{Theorem 6.}

Let $C_{4}$ be a cycle on 4 vertices. Then the total edge irregularity strength of the two copies of $C_{4}$ is

$$
\operatorname{tes}\left(2 C_{4}\right)=2 \operatorname{tes}\left(C_{4}\right)=4 \text {. }
$$

\section{Proof.}

Consider the cycle $C_{4}$ with the vertex set $V\left(C_{4}\right)=$ $\left\{u^{\mathrm{j}}: 1 \leq j \leq 4\right\}$ and the edge set $E\left(C_{4}\right)=\left\{u^{\mathrm{j}} u^{\mathrm{j}+1}: 1 \leq\right.$ $j \leq 3\} \cup\left\{u^{4} u^{1}\right\}$. It is proved in [8] that for cycle $C_{\mathrm{n}}$, $n \geq 3$,

$$
\operatorname{tes}\left(C_{n}\right)=\left\lceil\frac{n+2}{3}\right\rceil \text {. }
$$

\section{Thus tes $\left(C_{4}\right)=2$.}

Let $2 C_{4}$ be the disjoint union of two copies of $C_{4}$ with the vertex set $\mathrm{V}\left(2 C_{4}\right)=\left\{u_{i}^{j}: 1 \leq j \leq 4, i=1,2\right\}$ 
and the edge set $E\left(2 C_{4}\right)=\left\{u_{i}^{j} u_{i}^{j+1}: 1 \leq j \leq 3, i=1,2\right\}$ $\cup\left\{u_{i}^{4} u_{i}^{1}: i=1,2\right\}$. The inequality tes $\left(2 C_{4}\right) \geq 4$ holds by Theorem 4 . What follows is an edge irregular total labeling $g$ showing the converse inequality. Define the total labeling of $2 C_{4}$ as follows

$$
\begin{aligned}
& g\left(u_{i}^{j}\right)= \begin{cases}2 i-1, & \text { for } j=1,2 \text { and } i=1,2, \\
2 i, & \text { for } j=3,4 \text { and } i=1,2,\end{cases} \\
& g\left(u_{i}^{j} u_{i}^{j+1}\right)= \begin{cases}1, & \text { for } j=1,2 \text { and } i=1,2, \\
2, & \text { for } j=3,4 \text { and } i=1,2 .\end{cases}
\end{aligned}
$$

For edge-weights we have

$$
w t_{g}\left(u_{i}^{j} u_{i}^{j+1}\right)= \begin{cases}4 i+j-2, & \text { for } j=1,2 \text { and } i=1,2, \\ 4 i-j+5, & \text { for } j=3,4 \text { and } i=1,2\end{cases}
$$

One can check that all vertex and edge labels are at most 4 and the edge-weights successively attain values $3,4,5,6,7,8,9,10$, i.e. the edge-weights are distinct for all pairs of distinct edges. Thus the labeling $g$ provides that tes $\left(2 C_{4}\right)=2$ tes $\left(C_{4}\right)=4$.

\section{Conclusion}

In this paper we estimated the upper bound of the total edge irregularity strength of disjoint union of multiple copies of a graph. We proved that for a graph $G$ with $\operatorname{tes}(G)=k$, the total edge irregularity strength of the disjoint union of $m \geq 1$ copies of $G$ is at most $m k$. Moreover we showed that the upper bound of tes $(m G)$ is tight.

\section{Acknowledgement}

The research for this article was supported by KEGA 072TUKE-4/2014 2014.

\section{References}

[1] Ahmad A., Bača M., On vertex irregular total labelings, Ars Combin., vol 112, 2013, p. 129-139.

[2] Ahmad A., Bača M., Total edge irregularity strength of a categorical product of two paths, Ars Combin., vol 114, 2014, p. 203-212.

[3] Ahmad A., Bača M., Bashir Y., Siddiqui M.K., Total edge irregularity strength of strong product of two paths, Ars Combin., vol 106, 2012, p. 449-459.

[4] Ahmad A., Bača M., Siddiqui M.K., On edge irregular total labeling of categorical product of two cycles, Theory Comput. Syst., vol 54(1), 2014, p. 1-12.

[5] Amar D., Togni O., Irregularity strength of trees, Discrete
Math., vol 190, 1998, p. 15-38.

[6] Anholcer M., Kalkowski M., Przybylo J., A new upper bound for the total vertex irregularity strength of graphs, Discrete Math., vol 309, 2009, p. 6316-6317.

[7] Anholcer M., Palmer C., Irregular labellings of Circulant graphs, Discrete Math. vol 312, 2012, p. 3461-3466.

[8] Bača M., Jendrol' S., Miller M., Ryan J., On irregular total labellings, Discrete Math., vol 307, 2007, p. 1378-1388.

[9] Bača M., Lascsáková M., Siddiqui M.K., Total edge irregularity strength of toroidal fullerene, Math. Comput. Science, vol 7, 2013, p. 487-492.

[10] Bača M., Siddiqui M.K., Total edge irregularity strength of generalized prism, Applied Math. Comput., vol 235, 2014, p. 168-173.

[11] Bloom G.S., Golomb S.W., Applications of numbered undirected graphs, Proc. IEEE, vol 65, 1977, p. 562-570.

[12] Bloom G.S., Golomb S.W., Numbered complete graphs, unusual rules, and assorted applications, Theory and Applications of Graphs, Lecture Notes in Math. vol 642, 1978, p. 53-65.

[13] Bohman T., Kravitz D., On the irregularity strength of trees, J. Graph Theory, vol 45, 2004, p. 241-254.

[14] Brandt S., Miškuf J., Rautenbach D., On a conjecture about edge irregular total labellings, J. Graph Theory, vol 57, 2008, p. 333-343.

[15] Chartrand G., Jacobson M.S., Lehel J., Oellermann O.R., Ruiz S., Saba F., Irregular networks, Congr. Numer., vol 64, 1988, p. 187-192.

[16] Faudree R.J., Jacobson M. S., Lehel J., Schlep R.H., Irregular networks, regular graphs and integer matrices with distinct row and column sums, Discrete Math. vol 76, 1988, p. 223240.

[17] Frieze A., Gould R.J., Karonski M., Pfender F., On graph irregularity strength, J. Graph Theory, vol 41, 2002, p. 120137.

[18] Haque M.K.M., Irregular total labellings of generalized Petersen graphs, Theory Comput. Syst., vol 50, 2012, p. 537544.

[19] Ivančo J., Jendrol' S., Total edge irregularity strength of trees, Discussiones Math. Graph Theory, vol 26, 2006, p. 449-456.

[20] Jendrol'S., Miškuf J., Soták R., Total edge irregularity strength of complete and complete bipartite graphs, Electron. Notes Discrete Math., vol 28, 2007, p. 281-285.

[21] Jendrol' S., Miškuf J., Soták R., Total edge irregularity strength of complete graphs and complete bipartite graphs, Discrete Math., vol 310, 2010, p. 400-407.

[22] Kalkowski M., Karonski M., Pfender F., A new upper bound for the irregularity strength of graphs, SIAM J. Discrete Math., vol 25(3), 2011, p. 1319-1321.

[23] Majerski P., Przybylo J., Total vertex irregularity strength of 
dense graphs, J. Graph Theory, vol 76(1), 2014, p. 34-41.

[24] Majerski P., Przybylo J., On the irregularity strength of dense graphs, SIAM J. Discrete Math., vol 28(1), 2014, p. 197-205.

[25] Miškuf J., Jendrol' S., On total edge irregularity strength of the grids, Tatra Mt. Math. Publ., vol 36, 2007, p. 147-151.

[26] Nierhoff T., A tight bound on the irregularity strength of graphs, SIAM J. Discrete Math., vol 13, 2000, p. 313-323.

[27] Nurdin, Salman A.N.M., Baskoro E.T., The total edge-irregular strengths of the corona product of paths with some graphs, J. Combin. Math. Combin. Comput., vol 65, 2008, p. 163-175.

[28] Nurdin, Baskoro E.T., Salman A.N.M., Gaos N.N., On the total vertex irregularity strength of trees, Discrete Math., vol 310, 2010, p. 3043-3048.

[29] Nurdin, Baskoro E.T., Salman A.N.M., Gaos N.N., On the total vertex irregular labelings for several types of trees, Utilitas Math., vol 83, 2010, p. 277-290.

[30] Nurdin, Salman A.N.M., Gaos N.N., Baskoro E.T., On the total vertex-irregular strength of a disjoint union of $\mathrm{t}$ copies of a path, J. Combin. Math. Combin. Comput., vol 71, 2009, p. 227-233.

[31] Przybylo J., Linear bound on the irregularity strength and the total vertex irregularity strength of graphs, SIAM J. Discrete Math., vol 23, 2009, p. 511-516.

[32] Wijaya K., Slamin, Total vertex irregular labeling of wheels, fans, suns and friendship graphs, J. Combin. Math. Combin. Comput., vol 65, 2008, p. 103-112.

[33] Wijaya K., Slamin, Surahmat, Jendrol' S., Total vertex irregular labeling of complete bipartite graphs, J. Combin. Math. Combin. Comput., vol 55, 2005, p. 129-136. 